\title{
Observations of Comet 1884 Wolf
}

made at the U.S. N. Observatory with the 9.6 inch Equatorial, by Commander Wm. T. Sampson and Prof. E. Frisby. [Communicated by the Superintendent Commodore S. R. Franklin.]

\begin{tabular}{|c|c|c|c|c|c|c|c|c|c|c|}
\hline I 884 & Wash. M.'T. & $\Delta \alpha$ & $\Delta \delta$ & Comp. & Obs. & a app. & $\log p . \Delta$ & $\delta$ app. & $\log p .4$ & $*$ \\
\hline Sept. 2 I & $9^{\mathrm{h}} 3^{\mathrm{r}^{\mathrm{m}}} 9^{\mathrm{s}}$ & $-I^{m} \mathrm{I} 4.6 \mathrm{I}$ & + o' 19.9 & 20.4 & $\mathrm{~S}$ & $21^{\mathrm{h}}$ I $5^{\mathrm{m}} 53^{\mathrm{s}} \cdot 70$ & $8.65 \mathrm{I}$ & $+21^{\circ} 52^{\prime} 33^{\prime \prime} \cdot 3$ & 0.410 & I \\
\hline 22 & 104440 & + I 21.84 & -818.3 & 20.4 & $\mathrm{~F}$ & $21 \quad 16 \quad 21.85$ & $9 \cdot 3$ I I & +212422.0 & 0.455 & 2 \\
\hline Oct. & $\begin{array}{lll}8 & \text { I } 7 & 34\end{array}$ & to 29.62 & -4 & 20.4 & F & $\begin{array}{lll}21 & 25 & 44.36\end{array}$ & $8.385 n$ & +I 54644.8 & 37 & 3 \\
\hline 6 & ro $44 \quad 26$ & -1 $3^{8.54}$ & 一 I I & 20.4 & F & 2128 & 9.439 & +1 44449.7 & 0.592 & 4 \\
\hline 7 & $950 \quad 35$ & +142.66 & +84.3 & 20.4 & $F$ & $\begin{array}{lll}21 & 29 & 9.19\end{array}$ & & $73^{8.5}$ & 0.578 & 5 \\
\hline Nov. & $\begin{array}{lll}7 & 16 & 38\end{array}$ & - 048.27 & +554.6 & 20.4 & $\mathrm{~F}$ & $\begin{array}{lll}22 & 19 & 44.96\end{array}$ & 8.590 & $+\quad$ I 299.9 & 0.728 & 6 \\
\hline
\end{tabular}

Adopted mean Places of Comparison Stars.

\begin{tabular}{|c|c|c|c|}
\hline$*$ & ce 1884.0 & $\delta$ I 884.0 & Authority \\
\hline 1 & $21^{\mathrm{h}}$ I $7^{\mathrm{m}} \quad 4^{\mathrm{s}} \cdot 90$ & $+2 I^{\circ} 5 I^{\prime} 43^{\prime \prime} 6$ & BB. VI $+2 I^{\circ} 453 I^{\prime}$ \\
\hline 2 & $2 \mathrm{I}$ I 457.63 & $+2132 \quad 10.4$ & $W_{2} 2$ I $^{\mathrm{h}} 3$ I 9 \\
\hline 3 & $\begin{array}{lll}2 \mathrm{I} & 25 & \mathrm{II} .45\end{array}$ & +155021.3 & $W_{1} 21^{h} 55^{\circ}$ \\
\hline 4 & $\begin{array}{lll}21 & 29 & 38.85\end{array}$ & +145526.6 & $W_{1} 2 I^{h} 669$ \\
\hline 5 & $\begin{array}{lll}21 & 27 & 23.28\end{array}$ & $+r_{4} 94.4$ & $W_{1} 2 I^{h} 610$ \\
\hline 6 & $22 \quad 20 \quad 30.07$ & + I 2244.9 & $W_{1} 22^{h} 399$ \\
\hline
\end{tabular}

Observations de la Comète d'Encke (1885 ...)

faites à l'Observatoire de Lyon (Equatorial Brunner de 6 pouces) par M. Gonnessiat.

\begin{tabular}{|c|c|c|c|c|c|c|c|c|c|c|}
\hline I 885 & T. M. Lyon & $\Delta e$ & & $\Delta \delta$ & Vergl. & a app. & $\log p \cdot \Delta$ & $\delta$ app. & $\log p . \Delta$ & $*$ \\
\hline anv. 16 & $6^{\mathrm{h}} 5 \mathrm{I}^{\mathrm{m}} 53^{\mathrm{s}}$ & $-0^{\mathrm{m}} \mathrm{I} 7^{\mathrm{s}} \cdot 7^{8} 8$ & & - & 8 & $23^{\mathrm{h}} 10^{\mathrm{m}} 5^{8 .} \cdot 4 \mathrm{I}$ & 9.5 I I & -- & - & $\mathrm{I}$ \\
\hline I 6 & 7825 & - & + & $8^{\prime}$ I $5 . " \circ$ & 20 & 一 & - & $+4^{\circ} 57^{\prime}$ I $4^{\prime \prime} 0$ & 0.777 & I \\
\hline 18 & $7 \quad 1420$ & $\begin{array}{l}-0 \\
9.69\end{array}$ & & - & IO & 23 I3 27.94 & 9.549 & 一 & - & 2 \\
\hline 18 & $7 \quad 33 \quad 33$ & $\ldots$ & - & 733.9 & Io & 一 & 一 & $+575^{2.5}$ & 0.780 & 2 \\
\hline
\end{tabular}

Nébulosité très-pâle, avec faible condensation centrale. Comparaisons gênées ou interrompues par la brume.

Positions moyennes des étoiles de comparaison.

\begin{tabular}{|c|c|c|c|}
\hline$*$ & $\alpha \mathrm{I} 885.0$ & $\delta \mathrm{r} 885.0$ & Autorité \\
\hline $\mathbf{I}$ & $23^{\mathrm{h}}$ I I ${ }^{\mathrm{m}} \mathrm{I} 6.59-0.40$ & $+4^{\circ} 48^{\prime} 57^{\prime \prime \circ}+2.0$ & Sj. $95^{84-5}$ \\
\hline 2 & 23 I $3 \quad 38.03-0.40$ & $+5 \times 524.5+1.9$ & Sj. $960 \mathrm{r}$ \\
\hline
\end{tabular}

Comparaison des observations avec l'éphéméride de M. Backlund (Bull. astr.).

$$
(\mathrm{O}-\mathrm{C}) \text { : }
$$

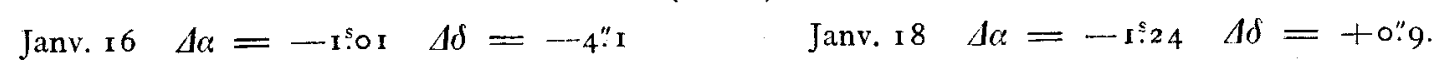

Lyon 1885 Janv. $2 \mathrm{I}$.

Ch. André. 